\title{
Las trayectorias de vida de los jóvenes de Santa Cruz de Tenerife en tiempos de crisis: una aproximación cualitativa
}

\author{
The Life Trajectories of Young People of Santa Cruz \\ de Tenerife in Times of Crisis: A Qualitative Approach
}

GOMER BETANCOR NUEZ y JOSUÉ GUTIÉRREZ BARROSO

Artículo recibido: 4 junio 2014

Solicitud de revisión: 4 noviembre 2014

Artículo aceptado: 19 enero 2015

Resumen

Este artículo, producto de un proyecto financiado por la Fundación Cajacanarias y la Asociación Cultural TuSantaCruz, analiza las perspectivas juveniles acerca de la realidad social, política y económica que marcan las diferentes trayectorias de vida de la juventud que habita en Santa Cruz de Tenerife. El uso del concepto de joven que hacemos en este trabajo es más abierto de lo habitual, pues concebimos que la juventud es una categoría social atravesada por cuestiones como el origen social, el nivel de estudios y la clase social, por lo que no es un colectivo caracterizado únicamente por un aspecto biológico-generacional. La metodología utilizada es de tipo cualitativa, concretamente se ha empleado la técnica denominada grupos de discusión.

Palabras Clave: juventud, trayectorias de vida, crisis, grupo de discusión, Santa Cruz de Tenerife

\begin{abstract}
This article, that was a product of a project funded by the Cajacanarias Foundation and the TuSantaCruz Cultural Association, analyzes the youth perspectives of the social, political and economic realities that affect at different life trajectories of youth that lives in Santa Cruz de Tenerife. Note that the use made of the concept of youth is more open than usual, because in this communication we conceive that youth is a social category traversed by issues such as social origin, educational level and social class, so this group is not only a group characterized by biological-generational relationships. The methodology used is qualitative, specifically we have used the technique called focus groups.
\end{abstract}




\section{INTRODUCCIÓN}

Este trabajo muestra los resultados de un proyecto de investigación desarrollado sobre los jóvenes en el municipio de Santa Cruz de Tenerife, titulado «Perspectivas de futuro de los jóvenes de Santa Cruz de Tenerife: nuevos retos, nuevas realidades» (Gutiérrez y Betancor, 2013). Este estudio, financiado por la Fundación Cajacanarias y gestionado por la Asociación Sociocultural TuSantaCruz, ha versado sobre uno de los temas más mediáticos de la actualidad, como es la afección de la situación económica, política y cultural de los jóvenes en una coyuntura de crisis como la existente en la actualidad.

Las tasas de desempleo juvenil están siendo muy elevadas, pues ascienden a un 55,49\% para España y aun 54,49\% para Canarias (INE, 2014). Esta juventud desempleada ve agravada su situación debido sobre todo a los recortes de las Administraciones Públicas, a la precarización de sus condiciones laborales y a la desprotección institucional que sufre este colectivo social, pues las administraciones son incapaces de atajar una situación socioeconómica de los jóvenes que se torna en dramática. En este sentido, Santa Cruz de Tenerife no es una excepción a este fenómeno de crisis económica. Dicho municipio, que contiene el 10\% de la población canaria y el 23\% de la población de la provincia de Santa Cruz de Tenerife, posee una tasa de desempleo juvenil similar a la de Canarias (ISTAC, 2013) teniendo además, como veremos en la investigación, ciertas peculiaridades que agravan aún más sus condiciones de vida.

La influencia de la crisis en los jóvenes es difícilmente cuestionable en las trayectorias de vida de los jóvenes de Santa Cruz de Tenerife, las cuales serán analizadas en este artículo. Así, a priori, podemos pensar que debido a la escasez y la precariedad en el empleo de los jóvenes, estos llegan a tener visiones pesimistas sobre su futuro a corto y a largo plazo, además de mostrar elevados niveles de desafección hacia las instituciones políticas locales, regionales y nacionales. Dichas hipótesis guiarán nuestra investigación a la hora de analizar las diferentes trayectorias vitales de los jóvenes en función de su situación personal, del contexto socioeconómico predominante (condiciones sociales), de su visión sobre la política y de sus expectativas. 


\section{ESTADO DE LA CUESTIÓN}

El término joven ha sido objeto de debate en la sociología debido a las diferentes concepciones asociadas al mismo, de las que derivan interpretaciones diferentes en los estudios sobre juventud. No es objeto de este artículo mostrar las diferentes posiciones teóricas sobre el concepto juventud, ya que excede con mucho el formato de este trabajo. Solamente diremos que después de una necesaria revisión bibliográfica, se observa, basándonos en Cardenal de la Nuez (2006: 3-44), la existencia de tres corrientes que conciben de forma muy diferente lo que es un joven: la empirista (Injuve, 2008, 2013), la crítica nominalista (Bourdieu, 2002; Martín Criado, 1998) y la transicional (Casal et al., 2006; Casal, 1996).

Para esta investigación, las aportaciones de estas corrientes serán fundamentales, intentando hacer una síntesis de todas ellas para una mejor conceptualización de la categoría joven. Evidentemente, es necesario catalogar al joven, por motivos pragmáticos a la hora de obtener datos, como la persona de entre 18 y 29 años, ya que normalmente la información ofrecida por las instituciones se basa en dicho intervalo de edad. Dicho sector puede tener algunos rasgos culturales propios, sobre todo relacionados con los valores y los itinerarios educativos, laborales y familiares. Sin embargo, no hay que sobrevalorar en lo que se refiere a las diferencias entre el universo valorativo juvenil y el de la sociedad en general, ya que las visiones de los jóvenes también pueden y suelen estar condicionadas por su posición en la estructura social.

Al margen de los estudios del Instituto de la Juventud Española (Injuve), donde se analiza la situación de los jóvenes y no tanto sus trayectorias de vida, existen gran cantidad de estudios sobre la transición desde el sistema educativo al mercado de trabajo, pues analizarla supone una «manera de comprender no sólo las dificultades y oportunidades experimentadas por los jóvenes en su transición a la vida activa, sino cómo una manera de hacer visibles los procesos de reproducción y cambio social» (Cardenal de la Nuez y Hernández, 2009: 34). En este punto también existen análisis acerca de la sobrecualificación de los jóvenes, que ha aumentado provocando en gran parte la pérdida de una parte importante de la ventaja salarial de los universitarios frente al resto de niveles educativos (García Montalvo, 2009). Por tanto, esos jóvenes «excesivamente cualificados» no son absorbidos por el sistema, estancándose en puestos de trabajos que requieren baja cualificación y que ofrecen salarios por debajo de lo que establece su nivel académico. 
También hay datos que indican que la situación de los jóvenes ya era precaria antes de la crisis. Así, ya en 2006, basándose en datos del Centro de Investigaciones Sociológicas, se afirmaba que «aumentaba de forma considerable el porcentaje de jóvenes que siguen ligados al sistema educativo más allá de los veinte años» y que «tanto el sistema educativo como el mercado laboral imponen a los jóvenes españoles periodos considerables de espera» (Mari Klose y Mari Klose, 2006: 8), que hacía que estuvieran un largo tiempo sin encontrar trabajo tras abandonar el sistema educativo. Por tanto, desde hace décadas, la frontera de separación entre juventud y edad adulta cada vez es más borrosa, pues ya no existen ritos de iniciación claros como la formación de la familia, la emancipación definitiva del hogar, tener un trabajo fijo, etc.

De esta forma, las generaciones jóvenes actuales «nacieron durante el boom económico, y se convierten en adultos en una etapa de incertidumbre económica» (De Miguel, 2006: 14). En este sentido, diversos estudios han mostrado que en todos los países de la ocDE un punto porcentual de desempleo adulto se asocia con un aumento de dos puntos de la tasa de paro juvenil (O’Higgins, 2011: 13). Por tanto, sí que existe cierta correspondencia entre la tasa de paro juvenil y la de los de más edad. En relación a las diferencias entre ambos grupos demográficos, si bien los jóvenes entran en el mercado laboral con una formación superior a la de los adultos, desde luego no tienen condiciones de trabajo similares a ellos. Esto es lo que se ha llamado la dualización del mercado de trabajo (Alaminos y Penalva, 2010: 188), la cual consiste en la sustitución del empleo estable de trabajadores con condiciones de trabajo dignas por trabajo precario y barato, desempeñado este último por mano de obra más joven.

Aún así, nunca hay que perder la perspectiva de que los jóvenes y el paro no existen en abstracto. Como afirma Martín Criado (1997), el origen social provoca que en la realidad no exista una tipología de joven, sino jóvenes con diferentes capacidades adquisitivas, niveles de estudios y posiciones en el mercado laboral, a los que lo único que une es la edad cronológica. De esta forma, para poder realizar análisis acerca de los jóvenes y la situación laboral, habrá que ser conscientes de que existen jóvenes de diferentes posiciones sociales.

Así, una salida a esa precarización laboral puede ser la economía sumergida, que si bien es tremendamente negativa para la economía del país, parece existir cierta tolerancia respecto a estas actitudes por parte de la población joven (vv.AA., 2011: 67). Por otro lado, la respuesta más habitual a esa precarización es el familismo, que viene a ser "un sistema de bienes- 
tar donde los problemas individuales de los integrantes de la red familiar (desempleo, falta de recursos y vivienda, enfermedad, etc.) tienden a ser definidos como asuntos familiares» (Mari-Klose y Mari-Klose, 2006: 150). Por lo tanto, la familia es el paraguas donde son protegidos los jóvenes en el plano sociolaboral.

Todos estos condicionantes económicos afectan a la percepción de la política por parte de los jóvenes. Es necesario superar la visión reduccionista sobre la relación entre jóvenes y política introduciendo una perspectiva más compleja de la situación, en la que tengan cabida tanto las nuevas condiciones en que los jóvenes viven hoy su juventud como la pluralidad de significados que confluyen en sus universos políticos. Esta cuestión fue magistralmente expuesta por Benedicto cuando, tras revistar los presupuestos teóricos y analíticos en los que se basan los diagnósticos negativos entre juventud y política, afirma a partir de evidencias que no hay que discutir sobre si la juventud actual está desenganchada de la política, si es escéptica o es alternativa, pues posiblemente se producen las tres cosas a la vez (Benedicto, 2008). De esta forma, la complejidad de la vida política juvenil no permite seguir manteniendo argumentaciones unidimensionales en ningún sentido, sino que es necesario comprender globalmente el fenómeno político en el colectivo juvenil.

\section{METODOLOGÍA}

La metodología escogida para esta investigación es de carácter cualitativo. Existen muchos referentes que podemos citar acerca de la metodología cualitativa (Vallés, 1997; Krueger, 1991; Bertaux, 2005; Alonso, 1998), pero pocos estudios de esta tipología en Canarias (Younis, 2000; Cardenal de la Nuez 2006). Es por ello que es pertinente un acercamiento cualitativo a las trayectorias juveniles en Canarias.

En nuestro estudio, la técnica de investigación utilizada ha sido el grupo de discusión, unas de las más apropiadas para recoger informaciones sobre la percepción de acontecimientos socialmente relevantes, así como para profundizar en los discursos que subyacen detrás de su comprensión y valoración (Ibáñez, 1986). Y es que el grupo de discusión viene a ser, en este sentido, un proyecto de conversación socializada en el que la producción de una «situación de comunicación grupal sirve para la captación y análisis de los discursos ideológicos y de las representaciones simbólicas que se asocian a cualquier fenómeno social»(Alonso, 1996: 62). 
Respecto al diseño muestral, en esta investigación se han respetado las cuestiones básicas relativas a la puesta en práctica de la técnica de grupo de discusión, tal y como recogen Ortí (1986: 181-183), Alonso (1998: 103) y Martin Criado (1997: 100). Los integrantes de cada grupo de discusión han sido jóvenes residentes en Santa Cruz de Tenerife desde hace al menos un año. La configuración de la muestra de este estudio se basó en la edad de los jóvenes, el nivel de estudios y la situación laboral, pues como se observó en el marco teórico referido al concepto de juventud, este no es un colectivo homogéneo, sino que está segmentado por variables como la clase social o el nivel de estudios. Por tanto, los grupos de discusión, quedaron distribuidos tal y como se recoge a continuación en la tabla I.

Tabla I.

Grupos de discusión de la investigación según diferentes variables sociodemográficas

\begin{tabular}{ll}
\hline G1 & $\begin{array}{l}\text { Jóvenes de } 25 \text { a } 29 \text { años con estudios de Bachillerato, FP Superior y } \\
\text { Universidad }\end{array}$ \\
\hline G2 & Jóvenes desempleados (prioritariamente de 25 a 29 años) \\
\hline G3 & $\begin{array}{l}\text { Jóvenes de } 18 \text { a } 24 \text { años con estudios de Bachillerato, FP Superior y } \\
\text { Universidad }\end{array}$ \\
\hline G4 & Jóvenes de 25 a 29 años con estudios hasta el Bachillerato, FP Medio \\
\hline G5 & Jóvenes de 18 a 24 años con estudios hasta la Eso, FP Medio \\
\hline
\end{tabular}

Fuente: elaboración propia

Como se observa en los estudios del Ijuve $(2012,2008)$, los análisis acerca de la juventud suelen incluir a los jóvenes de 15 a 17 años, cuestión que no se ha aplicado a esta investigación. Así, dicho sector juvenil ha sido excluido de nuestro estudio por varias razones. En primer lugar, por su baja representatividad respecto a los grupos de 18 a 29 años, pues dentro del colectivo juvenil, los jóvenes de 15 a 17 años son minoría.Además de dicho motivo existe otro de mucho mayor peso. De estos jóvenes, muy pocos habrán tenido experiencias laborales (pues la edad legal para trabajar es de 16 años y la mayoría de 16 y 17 años no habrá trabajado nunca, al menos de forma regulada) y sus niveles de estudios terminados serán, como mucho, de la Educación Secundaria Obligatoria (ESo) o Bachillerato. Así, los jóvenes de 15 a 17 tienen muchas menos probabilidades de realizar la transición de la escuela al mercado laboral, por lo que no son relevantes para esta investigación. 
Por otro lado, respecto a la configuración de los grupos de discusión, hemos distribuido a los jóvenes en dos intervalos etáreos, es decir, de diferentes grupos de edad -18 a 24 años y 25 a 29 años- porque planteamos que, en función de estudios de jóvenes analizados en el marco teórico, no será lo mismo un joven de 18 que uno de 29. Dicha segmentación por edad de la juventud también la avala el Centro de Investigaciones Sociológicas en sus análisis. Además, como se observa en el cuadro anterior, se consideró que en el intervalo de estudios superiores de 18 a 24 años se incluyen a los que tienen bachillerato, mientras que en el de 25 a 29 años a los de dicho nivel educativo se les incluyó en el intervalo de estudios más bajo. Esto se debe a que es un mérito para los de 18 a 24 años haber llegado a completar bachillerato -pues han tenido menos tiempo para estudiar-, mientras que para los de 25 a 29 quizás es un nivel bajo si pensamos que han tenido más tiempo para finalizar sus estudios. En cuanto al sexo, todos los grupos de discusión han sido mixtos, puesto que en las estadísticas no se aprecian diferencias excesivas entre sexos en la tasa de paro ni la de ocupación, si bien a nivel educativo tienen de media las mujeres una mayor formación (INE, 2014), razón por la cual hemos formado los grupos de mayores niveles formativos por una mayor cantidad de mujeres que de hombres. Por último, se decidió la creación de un grupo específico de jóvenes desempleados, dado el tremendo impacto de esta lacra social en la juventud santacrucera y canaria.

\section{RESULTADOS DE LA INVESTIGACIÓN}

Los resultados los hemos dividido en varias secciones para así poder analizarlos de una manera más clara para el lector. De esta forma, expondremos primeramente la visión de los jóvenes sobre el contexto general de su situación, para seguir con la percepción de la situación económica en Santa Cruz de Tenerife. Tras lo anterior, realizaremos el análisis de los puntos de vista de la realidad política de los diferentes jóvenes para terminar analizando las perspectivas de futuro de los mismos.

\subsection{Contexto general y vivencia de la situación}

Los distintos participantes parecen partir de la presunción de que el desempleo juvenil es una lacra generalizada, generándose un pragmatismo 
que se centra más en las consecuencias que en las causas de la misma. Se habla de la dificultad de encontrar empleo con la situación actual:

yo me acuerdo que antes se podía trabajar [sin problemas], tú decías que ibas a trabajar y te ponías a trabajar (Reunión de Grupo 4, en adelante RG).

Se relaciona también con la inestabilidad laboral que afecta a los jóvenes: «antes tenía más, siempre un trabajito de fin de semana, una semana y demás... Abora no» (RG 1). Se insiste en las pocas posibilidades de empleo, incluso en la economía sumergida:

antes, por ejemplo, $[\ldots]$ me salían trabajos de mozo de almacén, cosas que no son puestas por contrato, ese tipo de economía [...] sumergida al menos es una economía, pero es que ya ni eso (RG 1).

Esta sensación se puede corroborar con los datos oficiales, ya que según la Encuesta de Población Activa de 2012 del Instituto Canario de Estadística (ISTAC), la tasa de paro en el área metropolitana de Tenerife ha pasado de del 21,75\% en 2009 al 28.58\% en 2013. Ello afecta especialmente a los jóvenes, ya que según los datos del Injuve (2013), la tasa de paro juvenil en Canarias ha ido creciendo desde 2007 , pasando de un $16,3 \%$ ese año, a más de un $50 \%$ en la actualidad. Nada menos que prácticamente la mitad de los jóvenes en condiciones de trabajar en Canarias están desempleados, lo que genera un caldo de cultivo para el pesimismo.

Los procesos auto-reflexivos característicos de los grupos de discusión como técnica de investigación llevan a los participantes a construir socialmente este debate y proponer posibles hipótesis acerca de los motivos de su falta de inserción en el mercado laboral (Alonso, 1998; Conde, 2008). Como posible casuística, se apela a la falta de formación y cualificación para acceder a los puestos de trabajo: «también es culpa de nosotros al no estar bien cualificados dependiendo del puesto de trabajo» (RG 3). Esta última idea se relaciona con lo que ya planteaba Younis en estudios sobre jóvenes en Canarias de que el nivel de exigencia dentro de la juventud es bastante elevado, pues son muy poco autocomplacientes consigo mismos y con su grupo: "[los jóvenes] se critican muy duramente a sí mismos, como resultado de la culpa que han interiorizado y que les ha trasmitido la sociedad» (Younis, 2000: 664). Este sentimiento viene dado en una parte importante porque, sin hacerlo de modo explícito, los jóvenes manifiestan cuestiones relativas a la clase, origen social o características de la personalidad. Por ejemplo, es habitual que los jóvenes que trabajan y estudian di- 
gan a los que solamente estudian que se lo han dado todo hecho; que los que se quedan en casa y no se independizan son unos vagos, etc. Hay varios ejemplos en esta dirección en la investigación.

Otros participantes se sitúan en el extremo opuesto al exponer la existencia de sobrecualificación: "bay mucha gente preparada, universitaria, dos, tres carreras, idiomas, y tienen trabajos que ni siquiera antes babían pensado» (RG 4). Surge, por tanto, la idea de la sobrecualificación en este contexto, y la consecuencia de aumentar el paro de los titulados universitarios:

ahora mismo te ven el currículo y te ven que tienes un montón de cursos y un montón de carreras, y te contratan antes a uno que tenga un ciclo superior [...]. ¿Por qué? Porque al del ciclo superior le pagan menos que al que tiene la carrera universitaria» (RG 3).

Lo cierto es que como señala una de nuestras autoras referentes, desde hace unos lustros, en el contexto español «la inflación de titulados y la consecuente frustración de expectativas que ello conlleva es terreno abonado para la permanente crisis de nuestro sistema de enseñanza y el interminable debate sobre la relación entre educación y empleo» (Cardenal de la Nuez, 2006: 294).

Una vivencia compartida entre muchos participantes es la dificultad de llegar a fin de mes:

no solamente es la subida de los precios, sino la bajada de los sueldos, porque se ha quedado estancado. Eso es lo que [...] nos está afectando más (RG 3).

En algunos casos, sobre todo en los grupos con integrantes de clases medias-bajas, se habla de una coyuntura extraordinaria de impagos en algunas empresas:

En casa de mis suegros llevan tres meses sin cobrar, o sea cosas que no se veían, porque además son empresas sólidas en las que trabajan. [...]Y así está pero un montón... por lo menos en mi entorno, un montón de gente (RG 4).

Ello también es relacionado por algunos con la situación de las familias, con los despidos generalizados y con empresas que terminan cerrando:

gente de la familia que prácticamente se podía decir que tenían un trabajo fijo, ahora mismo $[\ldots]$ no saben si llegan a fin de año en ese trabajo porque están echando a un montón de gente del trabajo (RG 3). 
Esta concatenación de dificultades sociales nos lleva a particularizar a continuación la relevancia de la ayuda familiar.

En los grupos de discusión desarrollados la generalidad de los participantes habla de un palpable descenso del poder adquisitivo y, por ende, del nivel de vida de los habitantes de Santa Cruz de Tenerife (exceptuando los que viven en el centro de la ciudad), y el recurso a la familia como principal fuente de ayuda económica y hasta vital. De tal modo, se nota visiblemente un cambio en el nivel de vida, «una reducción de poder adquisitivo tremenda» que se refleja en necesidades básicas: "necesitas comprar [...] material escolar y no consigues eso porque claro, en tu familia las cosas están ya justas para comer y vestirte» (RG 2). Este descenso del nivel de vida se traduce en un empobrecimiento de la población que acrecienta las desigualdades sociales, señalándose que «la clase media también es la gran perjudicada» (RG 2).

El papel de la familia como colchón amortiguador del conflicto social se ha consolidado en un país como España, «que combina niveles muy altos de precariedad laboral con niveles de polarización y conflictividad social relativamente bajos. Este papel le viene otorgado, además de por razones que podríamos denominar culturales, por la propia estructura del sistema de protección social, que promueve la dependencia familiar» (Cardenal de la Nuez, 2006: 295). Es en este punto cuando sale a relucir la extraordinaria relevancia del papel de las familias como principal recurso de ayuda de los santacruceros, aunque algunos lo extrapolan a todo el país:

lo que está sosteniendo la paz social en España es la ayuda de [...] los familiares. O sea, si no existiera ese apoyo estaríamos en una situación muy muy grave (RG 2).

Este sistema informal de protección familiar implica una ayuda directa de los familiares y el sentimiento de protección por parte del integrante de la familia que necesita ayuda. Esto es y ha sido algo típico en España y se acentúa en Canarias, en gran parte debido a su tradicional nivel de paro estructural. No obstante, también nos encontramos ante lo que Cardenal de la Nuez denomina paradoja familista, según la cual esta red informal de protección reduce los riesgos que puede conllevar individualizar las dificultades económicas y sociales, pero en muchos casos sobrecarga de forma importante a las familias con menos recursos. Es en estos casos cuando el recurso al familismo se conecta más con el discurso de vivir al día.

Dejando claro esto, en un aspecto en especial se destaca el papel de las familias, que es al hablar del apoyo en el regreso a casa de los padres de muchos jóvenes que se habían emancipado o lo había intentado: 
«además del trabajo, $[\ldots]$ los que antes se habían lograr independizar después han tenido que volver a sus casas porque han perdido su trabajo» (RG 2).

En este sentido, cabe destacar que en el contexto español, ya con datos de 2007, se advertía que el acceso de los jóvenes a una vivienda estaba condicionado por la estructura residencial -caracterizada por los elevados precios de la vivienda-, por las condiciones económicas que se imponen desde el mercado laboral y los requisitos de las entidades financiadoras. Todo ello genera unos frenos estructurales que se imponen a los proyectos individuales, provocando en algunos casos un mayor o menor grado de exclusión residencial, línea que relaciona la emancipación económica con la residencial (Jiménez et al., 2008).

Además, estos cambios sociales son sufridos en carne propia por los jóvenes de Santa Cruz de Tenerife, que lo interiorizan con cierta resignación y frustración, que converge en un pesimismo generalizado:

se respira a lo mejor un poco un aire de pesimismo ¿no? Siempre que te encuentras con alguien [...], cuando no sabías qué decir, cuando hablabas del tiempo, ahora se habla de la crisis, todo el mundo está fatal (RG 1).

La consecuencia directa de ello es una perspectiva de vida cortoplacista donde la preocupación básica es la supervivencia del día a día:

yo y [...] mi pareja, más que vivir, es sobrevivir. Vivir con lo justo el día a día y intentar no pensar en el mañana [...] Intentamos no pensar y vivir el día a día como podamos (RG 3).

Hay que destacar que este discurso del familismo se acentúa especialmente en los grupos de participantes con estudios primarios y secundarios -clases medias-bajas-, en comparación a los grupos con integrantes de mayor nivel de estudios -clases medias-altas-.

Es de destacar que este discurso de ayuda familiar se conecta bastante bien con unas expectativas de sobrevivir, de vivir el día a día ya que esta acuciante situación no permite hacer muchos planes de futuro. Con ello entramos en el epígrafe siguiente, que es la conformación de las expectativas de futuro de la población joven santacrucera. 


\subsection{Percepción de la situación económica de los habitantes de Santa Cruz de Tenerife}

Los participantes de los grupos han relacionado esta situación de crisis económica con diversos factores, tanto económicos como políticos. Los discursos más representativos construidos en los grupos destacan la decadencia económica de la ciudad de Santa Cruz de Tenerife en los últimos años, el crecimiento de una economía sumergida y otras consecuencias derivadas de la agudización de la crisis.

En este sentido, se detecta un discurso de decadencia comercial y económica que viene afectando a Santa Cruz en los últimos años, algo que se comprueba por ejemplo paseando por la ciudad:

Santa Cruz ha perdido mucho porque ibas paseando los locales la Avenida de General Franco $[\ldots]$ y todos locales abiertos, con un montón de paseo, mucho tránsito.Vienes cuatro años acá, pasas por ahí, y hay más de [...] doce locales cerrados en ese tramo (RG 1).

Lo cierto es que en los grupos se reitera la imagen de Santa Cruz de Tenerife como ciudad de poca actividad: «se mantiene gracias al turismo yo que vivo en la zona centro por lo de cruceros y demás pero que ba bajado un montón» (RG 3). Otra participante señala en la misma dirección que Santa Cruz tiene «cada vez más tiendas cerradas, entonces si cierran [...] pues no entra dinero» (RG 2).

Sobre la posible casuística de la situación, algunas opiniones apuntan a la poca promoción de la economía a nivel local:

en Santa Cruz no se promueve casi nada, o sea tú vas a otros sitios y la gente suele salir a la calle un domingo, pero en Santa Cruz está muerto y a cierta hora no hay nadie (RG 1).

Esta situación de decadencia se compara en diversas intervenciones con La Laguna, que se alaba por su gestión de actividades e iniciativas:

a Santa Cruz la han dejado que la deriva la arrastrara y no han puesto de su parte para sacarla adelante, ahora están empezando a intentar otra vez dinamizar un poco Santa Cruz, pero yo pienso que van con mucho retraso», por lo que «se ha convertido más en capital cultural La Laguna que Santa Cruz (RG 2).

En este descenso de la actividad económica han influido factores generales del país que se han agudizado en el contexto de particular dependencia de la economía local, como son los siguientes: el derrumbe del sector inmobiliario y constructor; el corte del flujo crediticio desde la banca; los 
problemas de las administraciones públicas para financiarse; y el parón de la inversión pública y privada. Para comprender el descenso de la actividad de pequeñas y medianas empresas, se pueden observar datos por ejemplo del empleo registrado en el municipio, que ha descendido paulatinamente desde 2009 de casi 120.000 personas empleadas a poco más de 106.000 a finales de 2012 (Sociedad de Desarrollo, 2013: 7).

Un constante en los grupos a la hora de discutir la situación económica es su falta de profundización en aspectos propios. Los participantes lo asocian a su estrecha relación con factores políticos -mala gestión pública, corrupción política, etc.- y las consecuencias sociales de todo ello -empobrecimiento, aumento de desigualdades, aumento de delincuencia etc.Dicho esto, los temas que más destacan los participantes son los impagos de algunas empresas que generan desesperación en muchas familias y la economía sumergida como recurso necesario de supervivencia.

Asimismo, ha sido sorprendente la frecuencia del discurso de los impagos en empresas de las personas que viven en Santa Cruz de Tenerife -y su círculo de allegados- como una posible causa de la acentuación de la crisis económica. La situación económica se cataloga como bastante mala, retomando lo anteriormente comentado de impagos que dificultan una vida normal:

hay un montón de gente que está yendo a trabajar por no perder su puesto de trabajo porque no te quedan otras narices. [...] Porque no cobras prestación, ¿¿de qué vives? ¿Del aire (RG 3).

Esa precariedad y la mala situación laboral en forma de impagos o reducción de los ingresos afecta también a los autónomos, como declara un participante que se declara en dicha situación laboral:

Yo he tenido que [...] botar los precios. [...] Y la gente aún así te regatea:"no eso es muy caro señor mío". [...] No me estoy llevando yo nada, te bajo la mano de obra y aún así te pones a llorar $[\ldots . .$. . No voy a regalar mi trabajo tampoco (RG 3).

De otro lado, el afán de buscar diversas alternativas para minimizar al máximo los gastos se relaciona también con la cultura de la picaresca y la economía sumergida como problema social:

la corrupción está instalada desde el nivel más bajo. Yo nunca en mi vida he ido ni a un mecánico ni a un obrero que me diga si quiero la factura.Te lo preguntan directamente si la quiero con IGIC o sin IGIC, o sea, señores, eso ya es una esponja de corrupción instalada en el ADN de la población española (RG 2). 
Este posible problema de la economía sumergida sale en varias ocasiones como recurso y necesidad en una época en la que el adquisición de un trabajo formal es complicada, por lo que se concibe como una necesidad a corto plazo para subsistir:

yo entiendo que se tenga que perseguir porque sino estaríamos todos cogiendo debajo de la mesa como hay mucha gente. Pero es que hay mucha gente también [que cobra] la ayuda mínima (RG 4).

Se justifica en esa opción con el ejemplo de trabajos puntuales: «Me sale un cancamillo por abí ¿y le voy a decir que no? Vas y lo baces» (RG 3).Algo relevante de este discurso es que se palpa en diferentes temas, no solo cuando se trata la cuestión de la situación económica.Al tratar las expectativas de futuro de los jóvenes sale recurrentemente a colación, nuevamente, la economía sumergida como un recurso de subsistencia.

$Y$ es que como señalan estudios derivados de fuentes oficiales, la existencia de actividades económicas que no se reflejan en las estadísticas oficiales y que constituyen la economía sumergida es algo conocido, e incluso en gran medida, aceptado socialmente en España (vv. AA., 2011). Esa parte de aceptación e interiorización social es la parte más complicada de combatir, como se deduce de diversas intervenciones, ya que es una fuente básica de supervivencia.

\subsection{Diagnóstico político de Santa Cruz de Tenerife}

La mala gestión de la política económica es un tema que emerge con una especial fuerza discursiva en los grupos.Así, la crítica y desconfianza hacia la política local es una constante que se mantiene en todos los grupos y un tema siempre interrelacionado con los demás. Surge con una frecuencia muy grande la cuestión de la mala praxis en la política municipal, con una elevada desconfianza hacia la clase política: "[la ciudadanía] está hasta los buevos de los políticos y cada vez van menos a votar» (RG 1). Se apela así a la connivencia entre los políticos en la práctica institucional, tanto en el mismo partido como entre los partidos con representación:

[la gente] se siente engañada en general y al final dice mira pues esto al carajo y no voy, porque esta cansada porque a lo mejor votas a uno después se une con el partido no sé qué, de que tú no estas de acuerdo, y al final dices [...] ¿me están vacilando o qué? Al final pues la gente se cansa y no vota a nadie (RG 1). 
En este sentido, se critica también la imagen pública de los políticos, sobre todo por sus estrategias electoralistas:

yo lo que veo es que si cuando van a haber elecciones están ahí, que no se qué, que limpian todo, que tal [...]. Luego no hacen nada, porque luego pasan los seis meses después de que haya salido quien haya salido porque da igual quien salga (RG 4).

Cabe destacar la falta de estudios oficiales a nivel local sobre este tipo de cuestiones como la desafección hacia la política local, lo cual puede incluso ser concebido como un síntoma de dicho fenómeno ${ }^{1}$.

Asimismo, es interesante el hecho discursivo según el cual los participantes sitúan en dos paralelos distintos al pueblo y a los políticos, criticando la falta de compromiso con la realidad de éstos: «siempre pringamos los que no tenemos y los políticos no se quitan ellos de nada para que tengan los demás. O sea, yo sé que no vas a estar repartiendo por los barrios billetes, pero que puedes, creo yo, que puedes tomar medidas que ayuden a esa gente» (RG 4). Podemos relacionarlo con la situación general española en la que la desafección política muestra niveles escandalosos, en comparación a lustros anteriores «según la Encuesta Social Europea, España ha sido desde hace décadas el país con menos interés por la política de todos los europeos, incluyendo las nuevas democracias del este de Europa; el promedio de desinterés se ha movido en torno al $80 \%$ que declaraba que la política le interesa poco o nada. [...] Es cierto que la desafección política ha aumentado algo en estos últimos años, pero no tanto por la crisis económica como por la pasividad de los partidos ante la dramática situación del desempleo y los chalaneos ante los escándalos de corrupción», además de otros factores adyacentes (Montero y Torcal, 2013).

Esta desconfianza y la desafección política que en parte se deriva de la misma se ilustra con alguna crítica más específica, como la falta de transparencia política municipal: «hay una opacidad tremenda [...] Acceder a las cuentas públicas del Ayuntamiento de Santa Cruz para saber en que se invierte [...] es bastante complicado» (RG 2). La sensación que se extrae de la aplicación de los diferentes grupos es que en esta desconfianza generalizada en la política a nivel local, la corrupción política y la cultura del nepotismo tienen una incidencia directa. En esta línea, un tema amplia-

1 Siguiendo a estos autores, entendemos la desafección política como el desapego y/o alejamiento de ciudadanía de una democracia representativa con respecto a su sistema político, midiéndose generalmente por las percepciones de ineficiencia personal ante la política y los políticos, el cinismo hacia ambos y los sentimientos combinados de impotencia, indiferencia y aburrimiento hacia la política institucional (Montero y Torcal, 2006). 
mente compartido entre los santacruceros es la detección de prácticas corruptas en la política. Ello se relaciona constantemente con el nepotismo, que a nivel canario se plantea como práctica habitual y con los intereses propios de los políticos como grupo o clase específica, desarrollándose un discurso compartido inter grupalmente mediante el eje ellos-nosotros, un «ellos» que son los políticos, y un «nosotros» que vienen a ser los ciudadanos. En este sentido, cuando se habla de política, los discursos generan procesos de identificación proponiendo elementos en común entre diferentes grupos sociales y postulando un afuera constitutivo que cohesiona, por oposición, la unidad del conglomerado discursivo. De forma que, en este caso, definir a «ellos»-los políticos-, ayuda a reforzar un «nosotros» opuesto -la ciudadanía- (Laclau, 1990). Partir de ese eje ayuda a entender las implicaciones de los siguientes discursos de los participantes.

Hay una visión pesimista de la política en Santa Cruz de Tenerife. Se habla especialmente de la corrupción, en relación con la connivencia de tres grandes partidos dominantes a nivel local:

aparte de la corrupción que hay, el caciquismo y que un solo partido con sus coaliciones por lo que haga, aquí siempre esta Coalición Canaria. No sé cómo se lo montan, gana el pP y se ponen con el psoe, lo mismo los tres partidos lo mismo, lo único que ves Santa Cruz es tráfico influencias, corrupción (RG 3).

Se habla en este sentido de tráfico de influencias en la clase política para influir en su círculo de amistades:

tú hablas con gente que está metida a lo mejor en los ayuntamientos [...], y para estar aquí, si Pepito de tal, que conoce a no se quien y a no se cuanto y me metió. O sea, te lo dicen (RG 4).

En este sentido, la corrupción política en Canarias parece un fenómeno instalado en el imaginario colectivo. No en vano, según un estudio realizado por el Grupo de Investigación sobre Subdesarrollo y Atraso Social (GISAS) del departamento de geografía de la Universidad de La Laguna, Canarias registró entre los años 2000 y 2010 un total de 35 casos de corrupción y el 39,8\% de los municipios del archipiélago se vieron afectados, lo que representa el segundo mayor porcentaje por comunidades autónomas. ${ }^{2}$ A ello se suma que Canarias posee la tasa más elevada de casos de presunta co-

2 Disponible en: http://www.abc.es/local-canarias/20130622/abci-corrupcion-canarias-comunidades201306221351.html Consultado el 25 de Diciembre de 2013. 
rrupción investigados por los tribunales, según un informe del Consejo General del Poder Judicial divulgado recientemente ${ }^{3}$.

Asimismo, son muchos los participantes que señalan una relación de connivencia e influencia entre las élites económicas y las élites políticas a nivel local:

esa gente que tiene dinero son los que influyen en los políticos. En los políticos influyen ellos, ellos tienen un buen nivel socioeconómico y van a seguir votando a ese partido político (RG 4).

Se señala de modo similar que «esas personas tienen una condición política clara, y es así porque es la única forma de conseguir llegar a eso. O sea, tú puedes empezar siendo el más pobre de los pobres y trabajar como un cabrón y nunca vas a llegar a tener un nivel económico de una persona que al final se mete en política, y es lo malo (RG 3).

Estos intereses ya han sido mostrados por algunos estudios críticos sobre la relación entre intereses económicos y la política local en Canarias (vV.AA., 2009).

Se puede destacar por último la idea que surgió repetidamente en un grupo compuesto de personas de clases medias-bajas y con los integrantes de menor estudio ( $R G$ 5), donde se reconocía e incluso se justificaba la corrupción, saliendo un pesimismo antropológico que había permanecido latente durante el grupo. ${ }^{4}$ Es otra muestra de diferencias discursivas ligadas a posiciones sociales diferentes que apoyan la relativización del concepto de juventud (Martín Criado, 1998; Bourdieu, 2002).

\subsection{Expectativas de futuro}

Al cuestionar a los jóvenes del municipio sobre las posibles expectativas de futuro, el mensaje directo y habitual es que el futuro se ve "pésimo", «negro» $\mathrm{o}$ «con incertidumbre». Es sorprendente el consenso inter grupal que se genera con esta cuestión en un primer momento. Solo cuando se

3 «Las islas poseen la tasa más alta de casos de corrupción investigados por los tribunales». Disponible en: http://www.laopinion.es/canarias/2013/06/17/islas-poseen-tasa-alta-casos-corrupcion-investigados-tribunales/481772.html Consultado el 25 de Diciembre de 2013.

4 Aludimos a este grupo como compuesto de clases medias-bajas tomando como único indicador para ello el nivel de estudios (jóvenes de ambos sexos de 18 a 24 años con estudios hasta la Eso o FP medio), asumiendo el riesgo de caer en un sesgo de inferencia. Lo justificamos en que es un grupo de edad en la que se pueden tener estudios de mayor nivel. No contemplamos el grupo compuesto por desempleados como más propio de clases medias-bajas o clases medias descendentes porque es demasiado heterogéneo en edad y nivel de estudios, si bien el desempleo suele afectar en mayor medida a personas con un menor nivel de estudios (INE, 2014). 
profundiza más se implementa algún matiz según el grupo y se tratan más unos temas u otros, aunque esta tendencia general se mantiene. Se conecta especialmente el futuro pesimista a corto plazo con los participantes que dicen vivir al día, sobrevivir.

Como se ha esbozado ya, el enfoque en los jóvenes sobre su futuro es bastante pesimista. En algunos grupos sociales -los de clase media-baja- se conecta íntimamente con la necesidad de vivir al día. Pero este cortoplacismo también se refleja en la dimensión de unas necesidades básicas cubiertas pero un horizonte muy incierto, sobre todo por la gran dificultad de inserción laboral de los jóvenes.

En este contexto de gran crisis económica y social, los participantes señalan que de lo que se trata a corto plazo (pensamiento cortoplacista) es de la supervivencia:

-(Moderador) Y en este contexto, ¿cómo definimos buscarse la vida en el momento que estamos actualmente?

-Pues poner curriculum como un loco, buscar trabajo [...].

-Hacer lo que sea para sobrevivir.

-Y coger lo que está (no se entiende) por ahí.

-Porque te ofrecen porquerías pero tienes que entrar, porque dices es que no me queda de otra, mejor tener algo malo que nada. (RG 3).

Por ejemplo, en el grupo de jóvenes desempleados cuando se pregunta sobre el futuro a corto plazo, hay incluso más consenso sobre la mala situación:

[el futuro va a ser] súper negro, o sea es que aquí no vas a poder hacer nada por lo menos a corto plazo.Trabajo no vas a tener y menos de lo tuyo, imposible, y te vas a tener que ir fuera (RG 2).

En cuanto a una hipotética emancipación del hogar de los padres, se comenta la imposibilidad de acceder a la misma en la situación actual:

ahora todas las leyes que han sacado que favorecen al empresario en echarte [...] pagándote cuatro perras [...]. Con un sueldo ahora mínimo no puedes permitirte el lujo de salir de $[\ldots]$ casa tus padres (RG 2).

Es una máxima que se repite en diferentes intervenciones y cuyo espíritu es compartido por la mayoría, relacionándolo con la urgencia de vivir al día: "yo creo que vivimos el día a día y mañana igual será otro día, y pasado pues no sabes ya directamente» (RG 4). Ello implica una pura supervivencia sin plantearse el futuro a medio o corto plazo. 
Al tratarse las diferentes expectativas de futuro de los jóvenes santacruceros se ha cuestionado también sobre posibles alternativas que han ido surgiendo a lo largo de la dinámica grupal, es decir, indirectamente como vía más adecuada a la libre emisión de los discursos. De modo que las alternativas más discutidas han sido la necesidad de seguir formándose, donde los idiomas se ve como un elemento fundamental, y la alternativa de salir fuera.

Cuando hablamos de salir fuera nos referimos a salir de la isla de Tenerife, ya sea al resto de España (principalmente a la Península Ibérica) o al extranjero. Establecemos este matiz porque ha sido detectado por los participantes que por la situación excepcional de Canarias, en lo económico y geográfico, relacionan salir fuera con ir, por ejemplo, a vivir a Madrid. También en un grupo ha surgido la idea de emprender como posible alternativa, aunque en menor medida. La dificultad de encontrar un empleo en Tenerife hace que algunos de los participantes, sobre todo los que no son titulados ni poseen algún título de formación profesional, planteen la necesidad de seguir estudiando. Algunos de ellos además coinciden con un perfil de ciudadano que no desea emigrar, ya sea por déficits educativos o por cargas familiares. Una de las participantes señala así que:

cada vez te están exigiendo más y ya que no tenemos posibilidad de trabajar, yo creo que lo mejor es formarnos [...], ya sea para trabajar aquí o trabajar en cualquier otro país (RG 3).

La formación se plantea como necesidad para trabajar pero también para salir fuera, donde uno de los elementos clave para poder salir, en este caso de España, con los idiomas: «la gente está desesperada por aprender idiomas para irse» (RG 3).

No obstante, el tema más constante y del que más intensidad se habla es salir de Tenerife para buscar un empleo y tener mejorar las expectativas de futuro:

si a mí me ofrecen un puesto de trabajo en Madrid, yo cojo el primer avión y me voy, es decir, yo no voy a estar aquí perdiendo mi tiempo (RG 2).

Esta determinación para salir fuera, sobre todo al extranjero, se puede ver en un estudio de población general (no solo jóvenes), realizado por la Consultora de Recursos Humanos Adecco. Dicho estudio señala que entre 2008 y 2012 el número de canarios residentes en el extranjero ha crecido un 51,5\% al pasar de 72.000 a casi 110.000 personas, unas cifras que hablan por sí mismas. 
Es por ello que como alternativa de futuro muchos contemplan la emigración, ya fuera de España:

toda la gente que he conocido yo durante la carrera en estos años, el noventa por ciento está fuera (RG 3).

Y explican la alternativa a esta opción como necesidad básica y realista de encontrar empleo:

tú [...] tienes una formación, no tienes un puesto de trabajo, pues te tienes que ir fuera. [...] Yo tengo muchísimas amigas que se han ido [...] a Londres. Ha estado dos semanas eh buscando empleo, y a la tercera semana ya tienen trabajo. Y así un montón de gente (RG 4).

Se plantea como necesidad vital al no encontrar empleo en Tenerife y ver que las posibilidades de encontrarlo en un futuro tampoco son reales, viviéndose con resignación:

si en un tiempo de dos tres años no consigo nada por aquí emigraré también que voy hacer, hay que salir ¿no? (RG 3).

\section{CONCLUSIONES}

Después de exponer un análisis pormenorizado de los distintos hallazgos de la investigación, podemos proponer las conclusiones más relevantes del estudio y discutir los aspectos más destacados del mismo. Exponer estos hallazgos, algunos sorprendentes y otros más esperables, nos puede ayudar a una recapitulación del sentido de la investigación. En primer lugar, la vivencia de la realidad cotidiana viene marcada por unos acontecimientos vitales que han cambiado a partir de la crisis económica. La inestabilidad en el empleo, unas veces en forma de desempleo y otras veces en forma de precariedad laboral (temporalidad, trabajo a tiempo parcial, etc.), afecta de forma directa a la población santacrucera (especialmente a los jóvenes), que pierden poder adquisitivo, lo que implica un cambio sustancial en el nivel de vida. Como se ha visto a través del análisis de los grupos, ello es relacionado por los participantes con el clima de pesimismo social de la población del municipio. Se recurre, por tanto, al familismo como soporte principal de ayuda económica en las trayectorias de vida de la juventud. 
Es conveniente resaltar que no se ha detectado en la producción discursiva una especial diferenciación entre factores individuales y sociales a la hora de analizar las causas y consecuencias de la crisis económica. La razón principal de ello es que los participantes de los grupos asumen mediante una suerte de acuerdo tácito en que la crisis es algo que les viene dado, por lo que no cuestionan en profundidad sus causas y sí, como se ha visto, en sus consecuencias.

En cuanto a la dimensión política, los jóvenes se centran especialmente en el pesimismo detectado por los santacruceros en general. Entre los asuntos políticos que más preocupan destaca especialmente la percepción de una corrupción política generalizada difícil de combatir, saliendo a colación el discurso de la resignación que subraya la incapacidad de revertir la situación por parte del ciudadano. También se señala una connivencia entre los intereses económicos y los cargos políticos locales. En gran parte consecuencia de todo ello es la mala imagen que tienen los participantes de todos los políticos en general y la consustancial desafección y desconfianza hacia las prácticas de la clase política.

Todo este panorama y el diagnóstico político y social que hacen los jóvenes santacruceros influyen decisivamente en las pocas expectativas de futuro que ven en Santa Cruz de Tenerife, sobre todo a corto y medio plazo. Esta visión pesimista media en la idea de la emigración, tanto a la Península Ibérica como, sobre todo, al extranjero. Esta hipótesis sí la podemos confirmar ya que al tratarse esta cuestión, la mayoría del discurso grupalmente construido apuntaba a la necesidad de emigrar para encontrar un futuro mejor.

\section{BIBLIOGRAFÍA}

Adecco (2012): «Canarias es la Comunidad más afectada por la fuga de talento al exterior». Disponible en http://eldia.es/canarias/2012-01-24/8Canarias-es-comunidad-afectada-fuga-talento-exterior.htm. Consultado el 12 de Febrero de 2014.

Alaminos,A. y Penalva, C. (2010): "La vida cotidiana en la España del siglo XXI», en DEL CAmpo, S. y Tezanos, J. F. (2010): España. Una sociedad en cambio, Madrid, Biblioteca Nueva.

Alonso, L. E. (1998): La mirada cualitativa en sociología, Madrid, Fundamentos. 
Alonso, L.E. (1996), «El grupo de discusión en su práctica: memoria social, intertextualidad y acción comunicativa», Revista Internacional de Sociología, Tercera Época, 13, pp. 5-36.

Benedicto, J. A. (2008): «La juventud frente a la política: ¿desenganchada, escéptica, alternativa o las tres cosas a la vez?», Revista de Estudios de Juventud, 81, pp. 13-29.

BeRTAUX, D. (2005): Los relatos de vida. Una perspectiva etnosociológica, Barcelona, Bellaterra.

Bourdieu, P. (2002): «La juventud no es más que una palabra» en Bourdieu, P. (2002): Sociología y cultura, México, Grijalbo.

Cardenal De La Nuez, M. E. (2006): El paso a la vida adulta. Dilemas y estrategias ante el empleo flexible, Madrid, CIS.

Cardenal De La Nuez, M.E. y Hernández Guerra,A. (2009): «Abandono escolar, trabajo precario y reproducción social en Canarias: una aproximación empírica», Témpora, 12, pp. 19-36.

Casal, J. (1996): «Modos emergentes de transición a la vida adulta en el umbral del siglo veinte uno: aproximación sucesiva, precariedad y desestructuración», Revista Española de Investigaciones Sociológicas, 75, pp. 295-316.

Casal, J.; García, M.; Merino, R. y Quesada, M. (2006): «Aportaciones teóricas y metodológicas a la sociología de la juventud desde la perspectiva de la transición», en Papers, 79, pp. 21-48.

Conde, F. (2008): «Los grupos triangulares como espacios transicionales para la producción discursiva: un estudio sobre la vivienda en Huelva», en Gordo, A. y A. Serrano (2008): Estrategias y prácticas cualitativas de investigación social, Madrid, Pearson-Prentice Hall.

Consejo General Del Poder Jucicial (CGPJ) (2013): «Las islas poseen la tasa más alta de casos de corrupción investigados por los tribunales». Disponible en: http://www.laopinion.es/canarias/2013/06/17/islas-poseen-tasa-alta-casos-corrupcion-investigados-tribunales/481772.html Consultado el 25 de Diciembre de 2013.

De Miguel, J.M. (2006): «Prólogo» en Mari-Klose, P. y Mari-Klose, M. (2006): Edad del cambio. Jóvenes en los circuitos de solidaridad intergeneracional, cIs y Siglo XXI, Madrid.

García Montalvo, J. (2009): «El mercado laboral de los jóvenes en España»en Tezanos, J.F. (ed.):Juventud y exclusión social en España, Madrid, Sistema.

Grupo De Investigación Sobre Subdesarrollo Y Atraso Social (Gisas) (2013) «Canarias, segunda región con más casos de corrupción por número de mu- 
nicipios» Disponible en: http://www.abc.es/local-canarias/20130622/abci-corrupcion-canarias-comunidades-201306221351.html Consultado el 25 de Diciembre de 2013.

Gutiérrez, J. Y Betancor, G. (2013): Perspectiva de futuro de los jóvenes de Santa Cruz de Tenerife en un contexto de crisis: nuevos retos, nuevas realidades, Santa Cruz de Tenerife, Asociación TuSantaCruz.

IBAÑEZ, J. (1986): "Cómo se realiza una investigación mediante grupos de discusión«», en García-Ferrando M., Ibañez J. y Alvira, F. (1986): El análisis de la realidad social. Métodos y técnicas de investigación, Madrid, Alianza Editorial.

Instituto De La Juventud Española (InJuve) (2008): Informe Juventud 2007, Madrid, INJUVE.

Instituto De La Juventud Española (Injuve) (2012): Informe Juventud en España 2011, Madrid, INJUVE.

Instituto De La Juventud Española (injuve) (2013): Informe Juventud en España 2012, Madrid, INJUVE.

Instituto Nacional De Estadística (ine) (2014): Encuesta de Población Activa. Primer Trimestre 2014.

Jiménez, B.; Martín, A.; Navarrete, J.; Pinta, P.; Soler, R. y Tapia, A. (2008): La emancipación precaria. Transiciones juveniles a la vida adulta en España a comienzos del siglo XXI, Madrid, CIS.

KRUEGER, R.A. (1991): El grupo de discusión: guía práctica para la investigación aplicada, Barcelona, Pirámide.

LACLAU, E. (1990): New reflections on the revolution of our time, Londres, Verso.

Mari-Klose, P. y Mari-Klose, M. (2006): Edad del cambio. Jóvenes en los circuitos de solidaridad intergeneracional, Madrid, CIS y Siglo XXI.

Martín Criado, E. (1997): "El grupo de discusión como situación social», Revista Española de Investigaciones Sociológicas, 79, pp. 81-112.

Martín Criado, E. (1998): Producir la juventud, Madrid, Istmo.

Montero, J. R. y Torcal, M. (2006): Political disaffection in contemporary democracies, Londres, Routledge.

Montero, J. R. y Torcal, M. (2013): «No es el descontento, es la desafección», El País. Disponible en: http://elpais.com/elpais/2013/06/11/ opinion/1370978768_828729.html. Consultado en febrero de 2014.

O’Higgins, N. (2011): Desempleo juvenil y política de empleo, Madrid, Ministerio de Trabajo y Asuntos Sociales.

OrTí,A. (1986): «La apertura y el enfoque cualitativo o estructural: la entrevista abierta y la discusión de grupo» en García Ferrando, M.; IBAÑEz, J. y 
Alvira, F. (Comp.) (1986): El análisis de la realidad social. Método y técnicas de investigación, Madrid, Alianza.

Sociedad de Desarrollo de Santa Cruz de Tenerife (2013): Aspectos destacados de los indicadores básicos de coyuntura laboral de Santa Cruz de Tenerife, web: http://www.sociedad-desarrollo.com/es/Me-Interesa/Informacion-Socioeconomica/Datos-mercado-laboral/Documents/INDICADORES\%20B\%C3\%81 sicos\%20marzo\%202013.pdf. Consultado en diciembre de 2013.

VAllés, M. S. (1997): Técnicas cualitativas de investigación social, Madrid, Síntesis.

vV.AA. (2009): Expresión de libertad, Tenerife, Asociación Taller de Comunicación

- (2011): Dos ensayos de actualidad sobre la economía española, Madrid, FUNCAS.

Younis, J.A. (2000): Los jóvenes, como son y como son vistos, Las Palmas de Gran Canaria, Dirección General de Juventud del Gobierno de Canarias. 